\title{
Design of algorithm for detection of hidden objects from Tera hertz images
}

\author{
P.Vijayalakshmi ${ }^{1}$, M.Sumathi ${ }^{2}$ \\ ${ }^{I}$ (Department of Information Technology, Pandian Saraswathi Yadav Engineering College, Sivagangai \\ TN,India) \\ ${ }^{2}$ (Department of Computer Science, Sri Meenakshi Govt. Arts College for Women, Madurai, TN, India)
}

\begin{abstract}
An algorithm for detection of hidden objects from tera hertz images is presented. Presently, terahertz imaging employs object's radiometric temperatures in human to acquire images of concealed objects. But, it presents problem in temperature sensitive areas like oil and coal mines, factories etc. The aim of the paper is to detect and extract hidden objects underneath person's clothing. Here, a three stage approach is presented: In the first stage, edge based segmentation is applied after smoothing the image using bilateral filter. In the next stage, transform invariant shape descriptors, Gabor and gray level co-occurrence (GLCM) texture features of interested object regions are computed. Finally, a Euclidean distance criterion is used for classification. To appraise the technique, detection error and detection rate are calculated. Test results are compared with ground truth data obtained from the original image. Experiment results are found to be promising with 1.04\% detection error and $91.9 \%$ as detection rate. Potential applications in security include detection of weapons and explosive in public places like airports, stations etc.
\end{abstract}

Key words: Edge detection, GLCM features, Gobor features, Shape descriptors, THZ images.

\section{INTRODUCTION}

In the recent years, increased threats of criminal action have led to the development of many techniques for the detection of concealed weapons, explosives etc. They include metal detectors, X-ray scanners, and detection of explosive and are based on energetic radiation. Electromagnetic waves at Tera hertz frequencies are safe, penetrate barriers, have short wave lengths to allow discrimination between objects. They have unique reflection and absorption properties. Also, many explosives have characteristic signatures at terahertz wavelengths. A survey of object recognition/classification methods based on image moments was presented. They had reviewed various types of moments and moment-based invariants. They had studied the role for various image degradations and distortions that affects the shape descriptors for classification. They had reviewed numerical algorithms for moment computation in real applications [1]. Another paper had explained millimeter wave (MMW) sensors designed for detecting both metallic and nonmetallic objects placed on a human body and hidden under clothes. The sensor was based on the synchronized detection principle. It had estimated the power of back-scattered signal from hidden objects. Time-gating algorithm combined with threshold level was implemented to detect hidden objects at the distance [2].Detection and segmentation of concealed objects in Terahertz Image was presented. Standard segmentation algorithms were unable to segment or detect concealed objects. A two stage approach was presented. First, the noise from the image was removed using the anisotropic diffusion algorithm and then detected the boundaries of the concealed objects. A mixture of Gaussian model was used to study the distribution of the temperature inside the image to identify the concealed objects [3].A inter ferro metric imaging and sensing for the detection of explosives, weapons and drugs was explored. It worked with three objectives: (a) THz radiation to detect concealed weapons (b) to detect compounds such as explosives and illicit drugs that have characteristic $\mathrm{THz}$ spectra [4].Detection and identification of explosive RDX by Diffuse Reflection Spectroscopy was presented. The reflection spectrum of the explosive RDX was acquired from a diffuse reflection measurement using a THz time-domain spectroscopy system. Kramers-Kronig transform was applied over the reflection spectrum to obtain the absorption spectrum. The investigation demonstrated that $\mathrm{THz}$ technique could be capable of detecting and identifying hidden RDXrelated explosives [5].

The article was presented to provide a tutorial overview of developments in Tera hertz imaging. It was to detect concealed weapons from a standoff distance, especially where the flow of people could not be controlled. It' was a technological challenge that required innovative solutions in sensor technologies and image processing. A number of sensors based on different phenomenon as well as image processing techniques were to be developed to observe objects underneath people are clothing [6]. .A technology with a warning system was presented. It educated the customer about the use of technology and its applicability. It strongly recommended that potential customers to trial the technology in their own unique environments to determine the utility of this technology and for its adaptability to environmental pressures. The false alarms and missed detections that 
occurred were easily accommodated. [7]. Object detection is a critical part in many applications like image search, image understanding and scene analysis. However, still it is an open problem due to complexity of object classes and images. Current approaches used for object detection are top down and bottom up. Top down approaches include training stage and to define object configurations. The later approach includes low level image features to high level image features. Here, we have included both the approaches for detection of concealed objects [8],[9].Recent developments in the area of concealed weapon detection using electromagnetic methods including metal detection, magnetic field distortion, electromagnetic resonance, acoustic and ultrasonic inspection, millimeter waves, Terahertz imaging, Infrared, X-ray were reviewed. The advantages and disadvantages of various approaches were discussed. Research challenges were presented. Future research perspectives were in the above areas was analyzed [10].

A $35 \mathrm{GHz}$ imager was presented that was based on conical scan technology from low cost materials. In conjunction with an illumination chamber it was used to collect indoor images of people with weapons and illegal imports hidden under their clothing. That imager had a spot size of $20 \mathrm{~mm}$ and covered a field of view of $20 \times 10$ degrees that partially covered the body of knees to shoulders. A variant imager was designed and constructed. It had a field of view of $36 \times 18$ degrees and was capable of covering the whole body of an adult. That was achieved by increasing the number of direct detection receivers by implementing an improved optical design. The optics system consisted of a front grid, a polarization device which converted linear to circular polarization and a rotating scanner [11].THz time domain spectroscopy (THz-TDS) was presented. It was used to investigate explosives and establish a spectra database of explosive materials in the $\mathrm{THz}$ frequency range. Signatures of selected explosives and related compounds (ERCs) were identified in the $\mathrm{THz}$ band and maintained. THz spectra of ERCs were calculated based on Density Functional Theory [12]. The progress that had been made in Tera hertz technology over the years was reviewed. It identified the achievements, challenges and prospects in millimeter wave and terahertz technology for specialized screening tasks. It explored that many solids including explosives have characteristic spectroscopic signatures at terahertz wavelengths that were used to identify them [13].Detection of biological hazards using electronic Terahertz systems was presented. The discriminating sense of vulnerability to concealed threats by weapons or biological agents such as anthrax had led the scientific community to address the problem of detecting such concealed threats [14]. A survey of object recognition/classification methods based on image moments was presented. They had reviewed various types of moments and moment-based invariants. They had studied the role for various image degradations and distortions that affects the shape descriptors for classification. They had reviewed numerical algorithms for moment computation in real applications [15].

\section{PROPOSED MeTHOD}

Initially, images are processed to compensate for losses due to noise and other variations. In general, objects are featured in images by its color, edge, and shape and texture information. In this paper, three main attributes of object are considered at various spatial resolutions to detect any hidden object. First, the search image is divided into number of overlapping grids to reduce the missing probability. Both region-based and contour based shape descriptors are computed to distinguish shapes of different objects. Gobor, GLCM features are extracted as texture information to characterize objects. For robust detection, a combined feature vector of edge, shape and texture is employed. Test feature vector is calculated and compared with feature vectors of the search image using Euclidean distance classifier. To appraise the algorithm, detection rate and detection error are measured against ground truth data. Experiments are repeated both with combined and individual feature vector for gun, knife and needle images.

\subsection{Shape Features}

Shape descriptors are mathematical functions that are applied on images to produce numerical values. These numerical values are processed to provide information about objects. Two dimensional shape descriptors are of two categories, region-based descriptors and contour-based descriptors. Region-based descriptors characterize spatial distribution of pixel intensities and they include pixels in boundary and interior. Regionbased shape descriptors describe complex objects having multiple disconnected regions, simple objects with or without holes. Contour based shape descriptors are transform invariant and robust to noise. Moments invariants are more useful in shape analysis and they are used in distinguishing shape between different objects. A closed boundary is characterized by an ordered sequence $z(i)$ that represents the Euclidean distance between centroid and all $N$ bounding pixels of the digitized shape. Here, translation, rotation and scale invariant normalized contour sequence moment $\overline{m_{r}}$ and contour sequence central moment $\overline{\mu_{r}}$ are calculated for shape representation. Also, computation time is much less for spiral and concave shapes. Classification by using contour sequence moments comparatively good over area based moments. Shape descriptors $F_{1}, F_{2}, F_{3}$ are calculated using (2.1), (2.2) and (2.3). 


$$
\begin{gathered}
F_{1}=\frac{\left(\mu_{2}\right)^{\frac{1}{2}}}{m_{1}} \\
F_{2}=\frac{\mu_{3}}{\left(\mu_{2}\right)^{\frac{3}{2}}} \\
F_{3}=\frac{\mu_{4}}{\left(\mu_{2}\right)^{2}}
\end{gathered}
$$

\subsection{Texture Features}

$$
\begin{array}{cc}
F_{1}, F_{2}, F_{3} & - \text { Shape descriptors of regions } \\
m_{1} & - \text { First order moment } \\
\mu_{2}, \mu_{3}, \mu_{4} & \text {-- Second, third, fourth order central moments }
\end{array}
$$

A GLCM is a matrix where the number of rows and columns is equal to the number of gray levels, G, in a image. The matrix element $P(i, j \mid d, \theta)$ is the relative frequency with which two pixels, separated by distance $\mathrm{d}$, and in direction specified by the particular angle $(\theta)$. Here, gray level co-occurrence based texture features are computed in two steps. In the first step, pair wise spatial co-occurrences of pixels separated by a particular distance are charted by a gray level co-occurrence matrix (GLCM). Then using the GLCM, a set of texture features of interested object regions is computed. Here, entropy, correlation, energy, contrast and homogeneity features are computed. Energy content is computed using: (2.4) and is the sum of squared elements in the GLCM. Entropy represents the randomness of intensity distribution and is computed using: (2.5). Contrast is computed using: (2.6) that presents the amount of local variation present in the image. Correlation reveals the linearity present in the image and is measured using : (2.7). Homogeneity measures the closeness of the distribution of elements in the GLCM and is evaluated using: (2.8).

$$
\begin{gathered}
\text { Energy }=\sum_{m=0}^{G-1} \sum_{n=0}^{G-1} p(m, n)^{2} \\
\text { Entrophy }=\sum_{m=0}^{G-1} \sum_{n=0}^{G-1} p(m, n) \log p(m, n) \\
\text { Contrast }=\frac{1}{(G-1)^{2}} \sum_{m=0}^{G-1} \sum_{n=0}^{G-1}(m-n)^{2} p(m, n) \\
\text { Correlation }=\frac{\sum_{m=0}^{G-1} \sum_{n=0}^{G-1} m n p(m, n)-\mu_{x} \mu_{y}}{\sigma_{x} \sigma_{y}} \\
\mu_{x}=\sum_{m=0}^{G-1} m \sum_{n=0}^{G-1} p(m, n) \\
\sigma_{x}=\sum_{m=0}^{G-1}\left(m-\mu_{x}\right)^{2} \sum_{n=0}^{G-1} p(m, n) \\
\sigma_{y}=\sum_{m=0}^{G-1}\left(m-\mu_{y}\right)^{2} \sum_{n=0}^{G-1} p(m, n) \\
\text { Homogenity }=\sum_{m=0}^{G-1} \sum_{n=0}^{G-1} \frac{p(m, n)}{(1+m-n \mid}
\end{gathered}
$$

\section{Gobor Features}

Gabor filters have frequency and orientation representations similar to human visual system. They are are most appropriate for texture representation and for object discrimination. Results of symmetric and 
asymmetric Gobor filter are combined in a single quantity, called Gabor-energy. The Gabor-energy is closely related to the local power spectrum and it is associated with a pixel in an image. It is calculated as the squared modulus of the Fourier transform of the product between the image and a window function. Here, Gaussian window is used as a neighborhood function. Transformation is applied on the image with different orientations and scales. The resultant magnitude represents the energy $E(m, n)$ present in the image at different scales and is given: (2.9). From the magnitude, homogenous texture $\sigma_{m}$ is calculated using: (2.10) and (2.11).

$$
\begin{aligned}
& E(m, n)=\sum_{x} \sum_{y}\left|G_{m}(x, y)\right| \\
& \begin{array}{l}
m=0,1,2, \ldots \ldots \ldots . M-1 \\
n=0,1,2, \ldots \ldots \ldots \ldots . N-1
\end{array} \\
& \mu_{m}=\frac{E(m, n)}{P \times Q} \\
& \sigma_{m}=\frac{\sqrt{\sum_{x} \sum_{y}\left(\left|G_{m}(x, y)\right|-\mu_{m}\right)^{2}}}{P \times Q}
\end{aligned}
$$

\subsection{Algorithm}

$$
P \times Q-\text { imagesize }
$$

\section{Read search image;}

img=imread('humen.jpg');/ h

Divide the image into overlapping sub images

For each sub image,

function[X1,map1]=edg(R,R1);

Compute edge features;

function [X,map]=shpe(RGB,RGB1);

Compute Shape descriptors;

function $[\mathrm{X} 1, \mathrm{map} 1]=$ txtre1 $(\mathrm{R} 1, \mathrm{R} 2)$;

Compute GLCM feature

edg(img,img1);

shpe(img,img1);

txtre1(img,img1);

function $\mathrm{gb}=$ gabor_fn(sigma,theta,lambda,psi,gamma)

Compute Gabor features;

Compute entropy features

Compute a combined feature vector;

Train for gun images;

Train for knife images;

Train for needle images;

Create a feature database;

Read test image(gun/ knife/needle)

$\mathrm{R}=$ imread('gun.jpg');

R2=imread('1knife.jpg');

R3=imread('needle.jpg');

Create a test feature vector;

Classify using Euclidean criteria

\section{ANALYSIS OF EXPERIMENTAL RESULTS}

This paper has been investigated a feature based technique for detection of hidden objects from tera hertz images. It is implemented on Intel Dual Core Processor in Windows XP platform using MAT Lab 6.5. It is tested with images that consisted of gun, knife, needle etc. Here, edge features, shape descriptors, Gabor, GLCM texture features are computed to form a strong feature vector. Sample images used for search and test images in the experimentation are shown in figure 3.1 and 3.2. Since edges are less prone to noises, edge features alone are not sufficient to detect complex objects. Transform invariant shape descriptors and texture features are computed. GLCMs provide a quantitative picture of a spatial pattern of pixels. Pixels of same object have a higher correlation value compared to adjacent objects. Also, they are highly correlated with neighboring pixels than with distant pixels. Correlation co efficient is highly dependent on image window size. GLCM values are normalized to remove the image dependencies. Detection rate and accuracy are performance indicators for any 
detection algorithm. Experiments are conducted with individual feature vector and with combined feature vector. The following observations are made: Gradient energy is found to be larger near the edges and smaller in smooth areas of the image. Shape descriptors provide valuable information about intricate objects. Gobor features together with GLCM features provide robustness and stability to the system. Contour-based methods are sensitive to noise; region-based methods able to detect shape defections also. Pixel processing and connected component analysis are adopted to extract the object.

Experimental results are better for the combined feature compared to the individual feature vector. Detection error is found to be very less $0 \%$ for knife image. Similarly, detection error is $1.1 \%$ using combined feature than shape features for gun images. Detection errors for various images using combined feature vector are tabulated in Table 3.1., Table 3.2 and Table 3.3.Yet, overall performance is good for combined feature vector. An extensive search is carried out at various resolutions for multiple occurrences of same or different object. Due to limited availability of tera hertz images, we are able to conduct experiments only with a limited set of images that were taken from various scenes at different angles. For blurred and occluded images, detection error is poor. It is also found that detection rate is $94.4 \%$ for knife images and $88.8 \%$ for needle images. Computation of detection rate for various images is shown in Table 3.4. Experimental outputs for gun image are shown in figure 3.3.Experimental outputs for knife image are shown in figure 3.4. Experimental outputs for needle image are shown in figure 3.5.

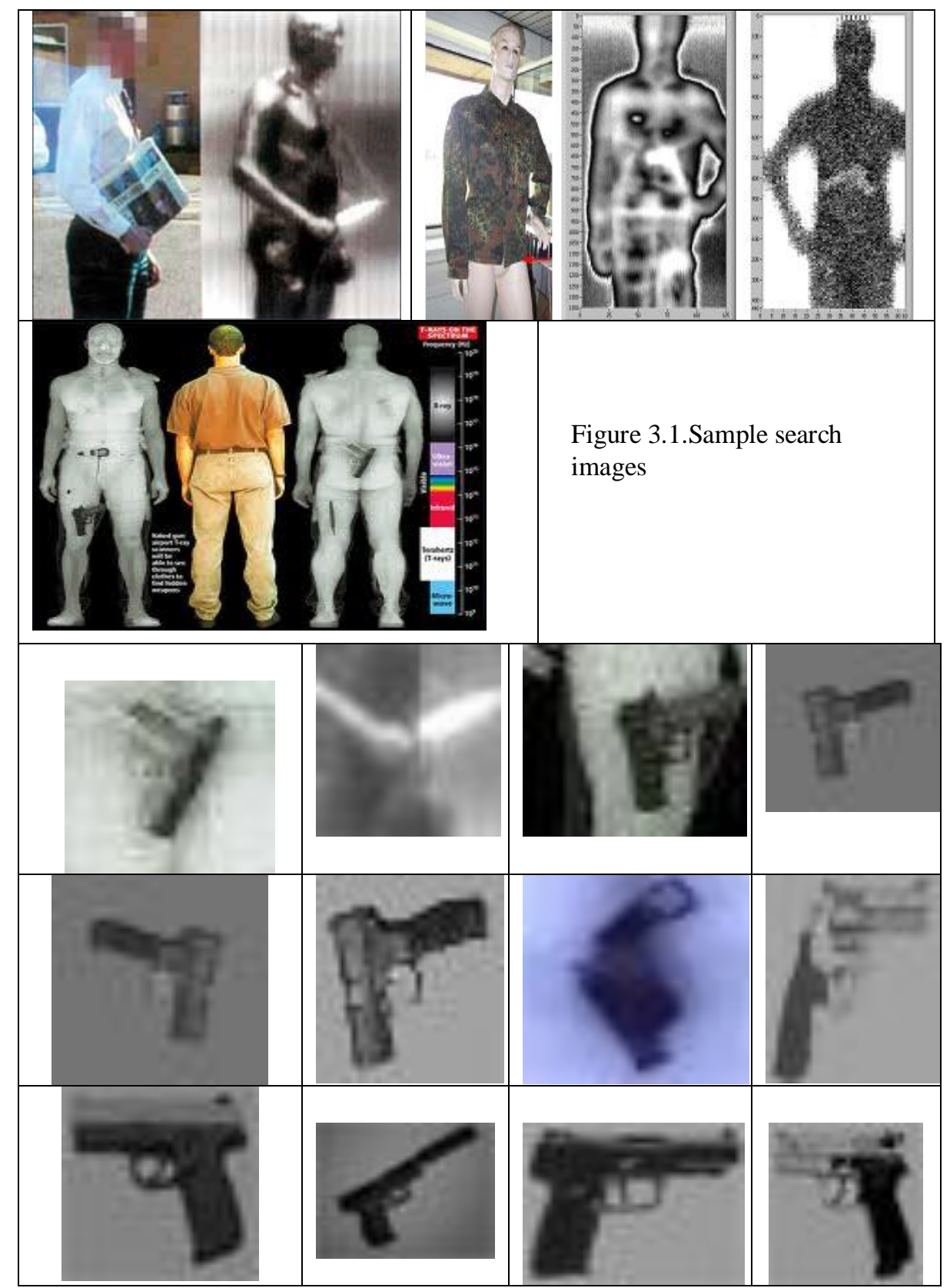

www.iosrjournals.org 


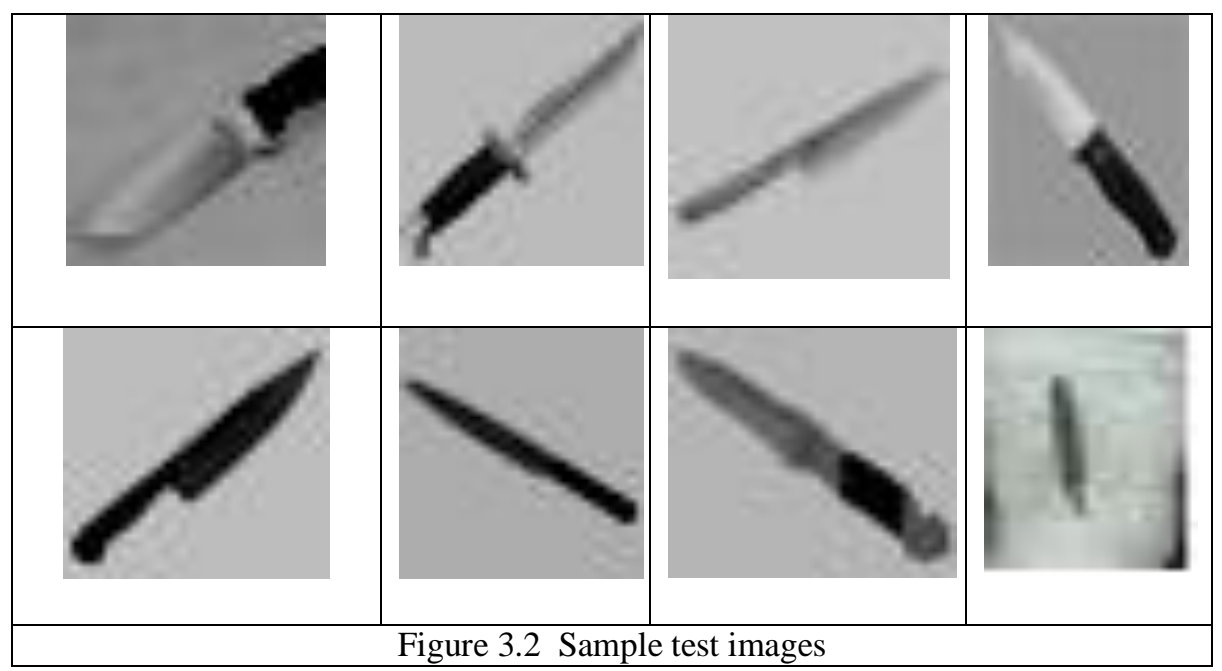

\begin{tabular}{|c|l|}
\hline INPUT HUMEN IMAGE & \multicolumn{1}{|c|}{ (b) } \\
\hline (a) & $\begin{array}{l}\text { Figure 3.3 Detection outputs for gun } \\
\text { a) Search image (190x275) } \\
\text { b) Combined feature } \\
\text { c) Shape feature } \\
\text { d) Texture feature }\end{array}$ \\
\hline (d) & \begin{tabular}{l} 
(a) \\
\hline
\end{tabular}
\end{tabular}

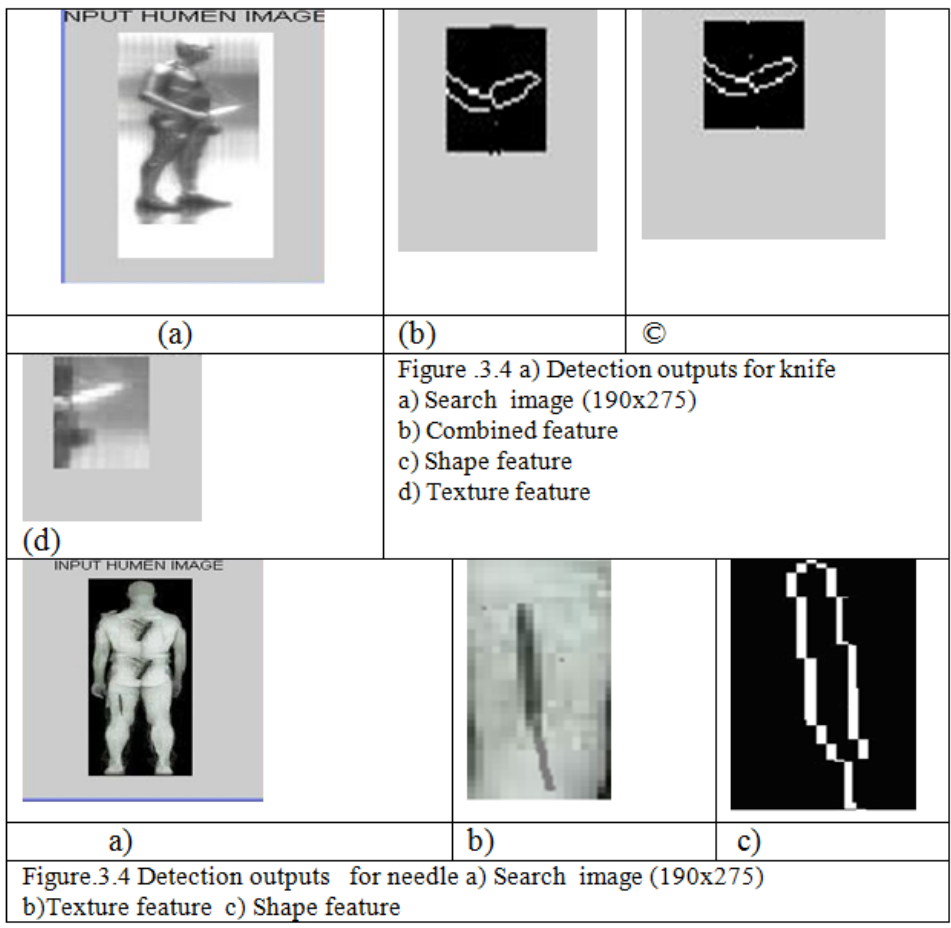




\begin{tabular}{|c|c|c|c|c|c|}
\hline \multicolumn{6}{|c|}{$\begin{array}{c}\text { TABLE } 3.1 \\
\text { Computation of Detection Error (Combined Feature) }\end{array}$} \\
\hline No & Test Images & Test Images & Actual pixels & $\begin{array}{l}\text { Estimated pixels } \\
\text { (detected) }\end{array}$ & $\begin{array}{l}\text { \%Detection error } \\
\text { E-A/Ax } 100\end{array}$ \\
\hline 1 & Gun & $32 \times 32$ & 91 & 90 & 1.1 \\
\hline 2 & Knife & $32 \times 32$ & 32 & 32 & 0 \\
\hline \multirow[t]{2}{*}{4} & Needle & $(24 \times 55)$ & 99 & 97 & 2.02 \\
\hline & & & & Average error & 1.04 \\
\hline
\end{tabular}

\begin{tabular}{|c|c|c|c|c|c|}
\hline \multicolumn{6}{|c|}{$\begin{array}{c}\text { TABLE } 3.2 \\
\text { Computation of Detection Error } \\
\text { For shape feature }\end{array}$} \\
\hline \multirow[t]{2}{*}{ S.No } & \multirow{2}{*}{ Test Images } & \multirow{2}{*}{$\begin{array}{c}\text { Image } \\
\text { Size }\end{array}$} & \multicolumn{2}{|c|}{ Shape features } & \multirow{2}{*}{$\begin{array}{c}\begin{array}{c}\text { \%Detection } \\
\text { error }\end{array} \\
\text { E-A/Ax } 100\end{array}$} \\
\hline & & & Actual pixels & $\begin{array}{l}\text { Estimated pixels } \\
\text { (detected) }\end{array}$ & \\
\hline 1 & Gun & $32 \times 32$ & 91 & 89 & 2.1 \\
\hline 2 & Knife & $32 \times 32$ & 32 & 32 & 0 \\
\hline 4 & Needle & $(24 \times 55)$ & 99 & 94 & 5.05 \\
\hline & & & & & 2.38 \\
\hline
\end{tabular}

TABLE 3.3

Computation of Detection error for Texture Features

\begin{tabular}{|l|l|l|l|l|l|}
\hline Sno & \multirow{2}{*}{ Test Images } & \multirow{2}{*}{ Test Images } & Texture features & \%Detection error \\
\cline { 4 - 6 } & & & Actual pixels & $\begin{array}{l}\text { Estimated } \\
\text { pixels } \\
\text { (detected) }\end{array}$ & E-A/Tx100 \\
\hline 1 & Gun & $32 \times 32$ & 315 & 310 & 1.58 \\
\hline 2 & Knife & $32 \times 32$ & 91 & 90 & 1.09 \\
\hline 4 & Needle & $(24 \times 55)$ & 220 & 211 & 4.09 \\
\hline \multicolumn{4}{|r|}{ Average } & 2.25 \\
\hline
\end{tabular}

\begin{tabular}{|l|l|l|l|l|l|}
\hline \multicolumn{6}{|c|}{ TABLE 3.4 } \\
\hline S,No & Test images & $\begin{array}{l}\text { Number of instances } \\
\text { tested }\end{array}$ & $\begin{array}{l}\text { Number of } \\
\text { Successful } \\
\text { detection }\end{array}$ & $\begin{array}{l}\text { Number of } \\
\text { Failures }\end{array}$ & $\begin{array}{l}\text { Detection } \\
\text { rate in \% }\end{array}$ \\
\hline $\mathbf{1}$ & Gun & 27 & 25 & 2 & 92.5 \\
\hline $\mathbf{2}$ & Knife & 18 & 17 & 1 & 94.4 \\
\hline $\mathbf{3}$ & Needle & 18 & 16 & 2 & 88.8 \\
\hline
\end{tabular}

\section{CONCLUSION}

This paper has explored the use of feature based technique for distinguishing interested regions in the sample tera hertz images. From the detection error, we have observed that combined feature vector makes the classification more stable and robust. Also, we have achieved $1.04 \%$ detection error for combined feature and 91.9\% detection rate from the experiments. Future work may consist of using additional metrics to compare the experimental results and testing the algorithm with different image sets. This new brand of detection system may enable more applications in security and safety.

\section{Journal papers:}

\section{REFERENCE}

[1]. Jan Flusser, Moment Invariants in Image Analysis, World Academy of Science, Engineering and Technology 11, 2005,pp:376381.

[2] KapilevichB.,Detecting Hidden Objects on Human Body Using Active Millimeter Wave Sensor, Sensors Journal, IEEE, Volume: 10, Issue: 11, On Page(s): $1746-1752$.

[3]. Xilin,Shencharles R Dietlein, Erich Grossman, Zoya Popvic and Francois G Meyer, Detection \& Segmentation of concealed objects in Terahertz Images, IEEE Transactions on Image Processing, 17 (12), pp 2465-2475, 2008

[4]. Federici, John F.; Schulkin, Brian; Huang, Feng; Gary, Dale; Barat, Robert; Oliveira, Filipe; Zimdars, David, THz imaging and sensing for security applications - explosives, weapons and drugs, ,Semiconductor Science and Technology, Volume 20, Issue 7, pp. S266-S280 (2005)

[5]. Hai-Bo Liu, Yunqing Chen, Glenn J. Bastiaans, and X.-C. Zhang, Detection and identification of explosive RDX by THz diffuse reflection spectroscopy, Optics Express, Vol. 14, Issue 1, pp. 415-423 (2006)

Magazine:

[6]. H. Chen, S. Lee, R. Rao, M.-A. Slamani, and P. Varshney, Imaging for concealed weapon detection," IEEE Signal Processing Magazine, pp. 52-61, March 2005. 
[7]. Dr Alan J. Lipton, Craig H. Heartwell, Dr Niels Haering, and Donald Madden, Critical Asset Protection, Perimeter Monitoring, and Threat Detection Using automated Video Surveillance, IEEE AESS systems magazine , 2003

Books:

[8]. Milan Sonaka, Vaclav Hlavac, Roger Boyle, Image processing, Analysis, and Machine Vision (Thomson learning Inc. ISBN : 981240-061-3, 1998).

[9]. Rafael C.Gonzalez, Richard E.Woods, Digital Image processing ( PHI Learning Private Ltd. New Delhi, ISBN: 978-81-203-36407. 2008).

\section{Proceedings:}

[10]. A. Agurto, Y. Li, G. Tian, N. Bowring, and S. Lockwood, "A review of Concealed weapon detection and research in perspective," in Proceedings of the 2007 IEEE International Conference on MonE02 Networking, Sensing and Control, 2007, pp. 443- 448.

[11]. Appleby, Roger; Anderton, Rupert N.; Price, Sean; Sinclair, Gordon N.; Coward, Peter R. , Whole-body 35-GHz security scanner, Radar Sensor Technology VIII and Passive Millimeter-Wave Imaging Technology VII.Proceedings of the SPIE, Volume 5410, pp. 244-251 (2004).

[12]. Yunqing Chen, Haibo Liu, X.-C. Zhang, etc, "Spectroscopic characterization of explosives in the far infrared region", Proceedings of SPIE, 5411, pp1-7, 2004

[13]. Michael C Kemp, Millimetre Wave and Terahertz Technology for the Detection of Concealed Threats - A Review, , Iconal Technology Ltd, St John's Innovation Centre, Cambridge, United Kingdom, Optics and Photonics for Counter-Terrorism and Crime Fighting II Proc. of SPIE Vol. 6402, 64020D, · 0277-786X/06/\$15 · doi: 10.1117/12.692612, 2006

\section{Reports:}

[14]. Peter Baines, Hazardous Materials: Chemical Biological Radiological and Nuclear - A Review: 2004 to 2007, Detective Report -, National Institute of Forensic Science, Australia.

[15]. Wai Lam Chan, Jason Deibel and Daniel M Mittleman, Imaging with terahertz radiation, IOP Publishing, Reports On Progress In Physics, Rep. Prog. Phys. 70 (2007) 1325-1379, doi:10.1088/0034-4885/70/8/R02. 